\title{
Una aproximación teórica para la aplicación de la metodología del enfoque mixto en la investigación en enfermería
}

\section{A theoretical approximation for the application of a mixed approach when conducting a research in the area of Nursing}

\section{ISSN 2071-8748 \\ E-ISSN 2218-3345 \\ (c) (1) (2) \\ BY NC SA}

DOI: https://doi.org/10.5377/entorno.v0i69.9562

URI: http://hdl.handle.net/11298/1156
María Sofía Albayero https://orcid.org/0000-0003-1876-9179

Marisol Tejada-Hernández https://orcid.org/0000-0001-5406-3427

Juana de Jesús Cerritos https://orcid.org/0000-0003-4391-7081 Investigadoras del Instituto Tecnológico Escuela Técnica para la Salud

Recibido: 14 de febrero 2020 Aceptado: 27 de junio de 2020

\section{Resumen}

El propósito de este artículo es hacer una aproximación teórica de la metodología del enfoque mixto en la investigación en enfermería de forma ilustrativa, como un primer aporte a la discusión de la temática.

Desde un punto de vista general, son cuatro los conceptos fuertemente ligados al quehacer investigativo de la enfermería: la persona, el entorno, la salud y el cuidado (Santos Heredero, Rodríguez Arias, \& Rodríguez Ballestero, 2004).

El propósito de la investigación mixta no es sustituir a ninguno de los enfoques que la componen, sino que, por el contrario, su meta es utilizar las fortalezas de

\section{Abstract}

The purpose of this article is to make a theoretical approximation on the mixed approach used when doing Nursing research in an illustrative manner, as a first contribution to the discussion of this topic.

From a general point of view, there are four concepts which are strongly linked to the investigative work on Nursing: the individual, the environment, health, and care (Santos Heredero, Rodríguez Arias, \& Rodríguez Ballestero, 2004).

The purpose of a mixed approach is not that of replace any of the approaches that make it up, but, on the contrary, that of utilizing the strengths of both types of 
ambos tipos de indagación, combinándolas y tratando de minimizar sus debilidades potenciales (Lara Muñoz, 2011).

La enfermería como profesión se ha configurado con capacidad y responsabilidad para desarrollar investigaciones científicas que proporcionen la capacidad de resolver necesidades urgentes en el ámbito de la salud, lo presentado hasta el momento es una apertura de la discusión.

\section{Palabras clave}

Enfoque mixto de investigación; Metodología científica; Enfermería - Investigaciones; Investigación cualitativa; Investigación cuantitativa.

\section{Introducción}

En principio cabe enfatizar que un proyecto de investigación demanda un trabajo intelectual ordenado, en virtud de los razonamientos lógicos necesarios más que de una gran cantidad de medios para desarrollarlo; en concreto, se necesita ser creativo.

La investigación es un medio importante para el desarrollo de una sociedad y de igual forma para el área de la salud. El propósito de este artículo es hacer una aproximación teórica de la metodología del enfoque mixto en la investigación en enfermería de forma ilustrativa como un primer aporte a la discusión de la temática propuesta.

\section{Del método hacia el diseño metodológico}

La investigación es un proceso que se emprende con el planteamiento del problema, que es esencial solucionar, para ello se requiere construir un diseño metodológico que permita llegar a descubrir, explicar y, si es posible, predecir probabilísticamente o en términos de tendencia determinadas situaciones, o dar respuestas a partir de la documentación de las cualidades que presenta dicho problema (Rojas Soriano, 2006;Hernández Sampieri, Fernández Collado, \& Baptista Lucio, 2014).

Comprendiendo que el método, desde el plano filosófico, es la vía más racional para aproximarnos a la verdad investigation, by combining them and trying to minimize their potential weaknesses (Lara Muñoz, 2011).

Nursing as a profession has been drafted with the capacity and responsibility to develop scientific research which product can then provide the capacity for solving urgent needs in the field of health; what has been already presented is a starting point for this discussion.

\section{Keywords}

Mixed approach; Scientific Method; Nursing - Research; Qualitative research; Quantitative research.

(Núñez Tenorio, 1989); y que el método científico, por lo tanto, es el camino que se sigue en la investigación a través de procedimientos concebidos para descubrir, generalizar y profundizar los conocimientos para demostrarlos rigurosamente (De Gortari, 1979), de modo que la metodología son las diferentes fases de ejecución y coordinación eficiente de tiempo y recursos en el diseño de una investigación científica.

Figura 1. Abstracción del diseño metodológico de investigación, originado por el método, pasando por el método científico y convirtiéndose en un proceso metodológico como paso final (Plantilla tomada de freepik.es y adaptada por las autoras como elaboración propia)

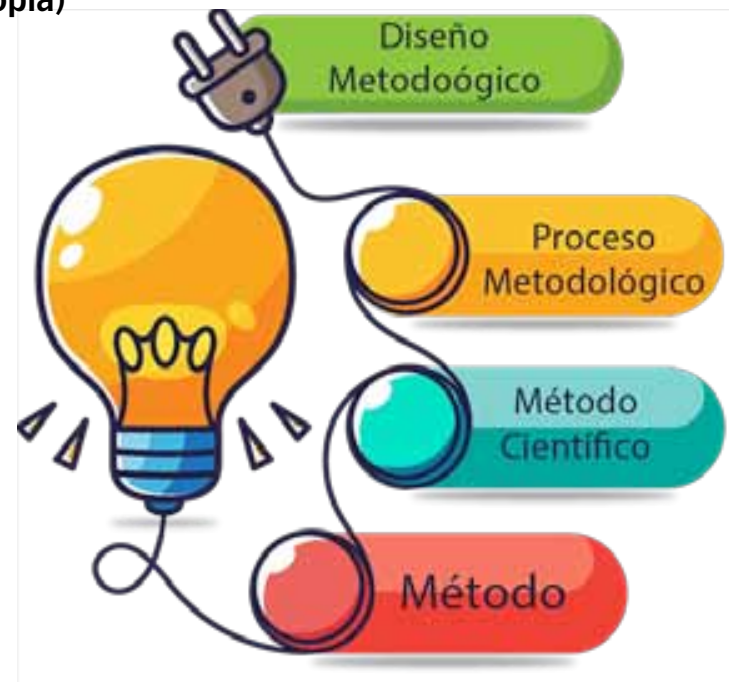


Avanzando en el abordaje, el diseño metodológico de una investigación es un procedimiento reflexivo permanente sobre la pertinencia de las opciones que adopta el investigador en relación con las particularidades de su ejercicio científico. Asimismo, es un proceso sistemático y metódico, puesto que posibilita aplicar un conjunto de reglas aceptadas para construir y validar el conocimiento obtenido a través de un sistema más amplio que le da sentido a la totalidad de las acciones realizadas previamente planificadas. Además, es un proceso controlado y de carácter replicable, ya que supone que el conocimiento obtenido puede ser repetido un sinnúmero de veces por otros investigadores.

\section{Del enfoque del número al enfoque de la cualidad}

Adrián Paensa, en su libro Matemática para todos, expresa que "la matemática es una usina (instalación industrial) constante y consistente de problemas que parecen atentar contra la intuición. Pero, justamente, al pensarlos uno se educa, se entrena y se prepara porque la experiencia demuestra que es muy posible que vuelvan a aparecer en la vida cotidiana usando disfraces mucho más sofisticados" (Paensa, 2012, p. 7).

Por su parte, lo fundamental del enfoque cuantitativo es la medición y estimación de las magnitudes de los fenómenos o problemas de investigación, y se pregunta cada cuánto ocurren y con qué magnitud Es decir, que la información recolectada la fundamenta en la medición de las variables o conceptos contenidos en las hipótesis, en el sentido estricto de que se puedan probar, y que se lleva a cabo al utilizar procedimientos estandarizados y aceptados por una comunidad científica. Para que una investigación sea creíble y aceptada por otros investigadores (Hernández Sampieri, Fernández Collado y Baptista Lucio, 2014).

Otro de los fundamentos importantes del enfoque cuantitativo, es el apoyo en la estadística, que le permite la utilización de diversas técnicas según sea la necesidad del tipo de estudio y lo que se pretende cuantificar (Hurtado de Barrera, 2012). Mientras que el enfoque cualitativo se sitúa en cualificar y describir las problemáticas de investigación a partir de rasgos determinantes, según sean percibidos por los elementos mismos que están dentro de la situación estudiada (Lara Muñoz, 2011).

Por otra parte, el enfoque cuantitativo explica los problemas de investigación mediante el método hipotético deductivo para justificar las hipótesis planteadas, transitando de lo general a lo particular. Mientras que el cualitativo lo hace por medio del razonamiento inductivo para comprender dichos problemas desde las experiencias y puntos de vista de los actores sociales sobre lo que dicen que saben y lo que realmente hacen (Izcara Palacios, 2014).

Figura 2. Abstracción de la conjugación de los enfoques de investigación cuantitativo y cualitativo (Plantilla tomada de freepik.es y adaptada por las autoras como elaboración propia)

\section{Enfoque Mixto}

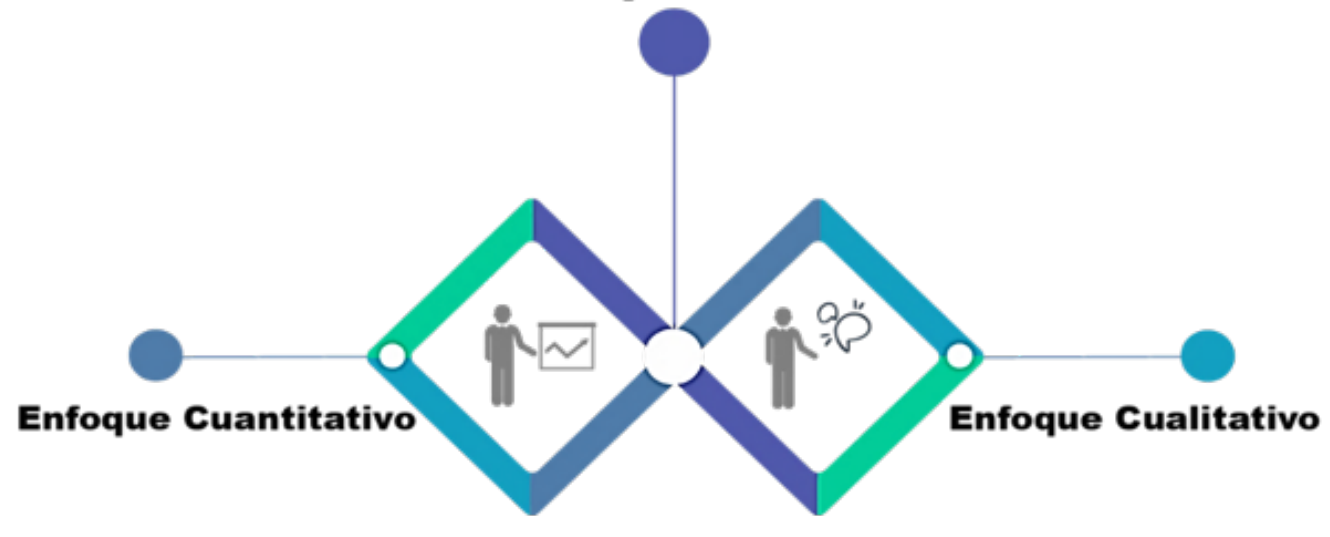


Tomando en cuenta que ninguno de los enfoques anteriormente descritos es mejor, no deberían ser vistos como polos opuestos. La combinación de ambos, es lo que los especialistas denominan como enfoque mixto en la investigación (Hernández Sampieri, Fernández Collado y Baptista Lucio, 2014; Creswell, 2009).

3. Propuesta del enfoque mixto: dos metodologías para el desarrollo de la investigación en enfermería; una discusión en proceso

La preparación y experiencias propias del investigador intervienen discrecionalmente en su decisión de desde qué enfoque plantearse el diseño metodológico de su investigación (Creswell, 2009). Una de las áreas de desempeño de los profesionales de enfermería es la investigación. Por supuesto que, dependiendo del nivel de formación, aplican en el nivel de tecnólogo y licenciatura.

En los últimos años, la enfermería va abriendo brecha en la investigación científica y se trazan algunos de los muchos campos en los que esta juega un papel investigador fundamental, por lo que está en disposición de plantear una metodología precisa para desarrollar un trabajo de investigación holístico, en lo que a salud se refiere. Desde un punto de vista general, son cuatro los conceptos fuertemente ligados al quehacer investigativo de la enfermería (ver figura 3).

Figura 3. Conceptos ligados al quehacer investigativo de la enfermería, con base en el planteamiento de Santos Heredero, Rodríguez Arias, Rodríguez Ballestero, 2004. (Plantilla tomada de freepik.es y adaptada por las autoras como elaboración propia)

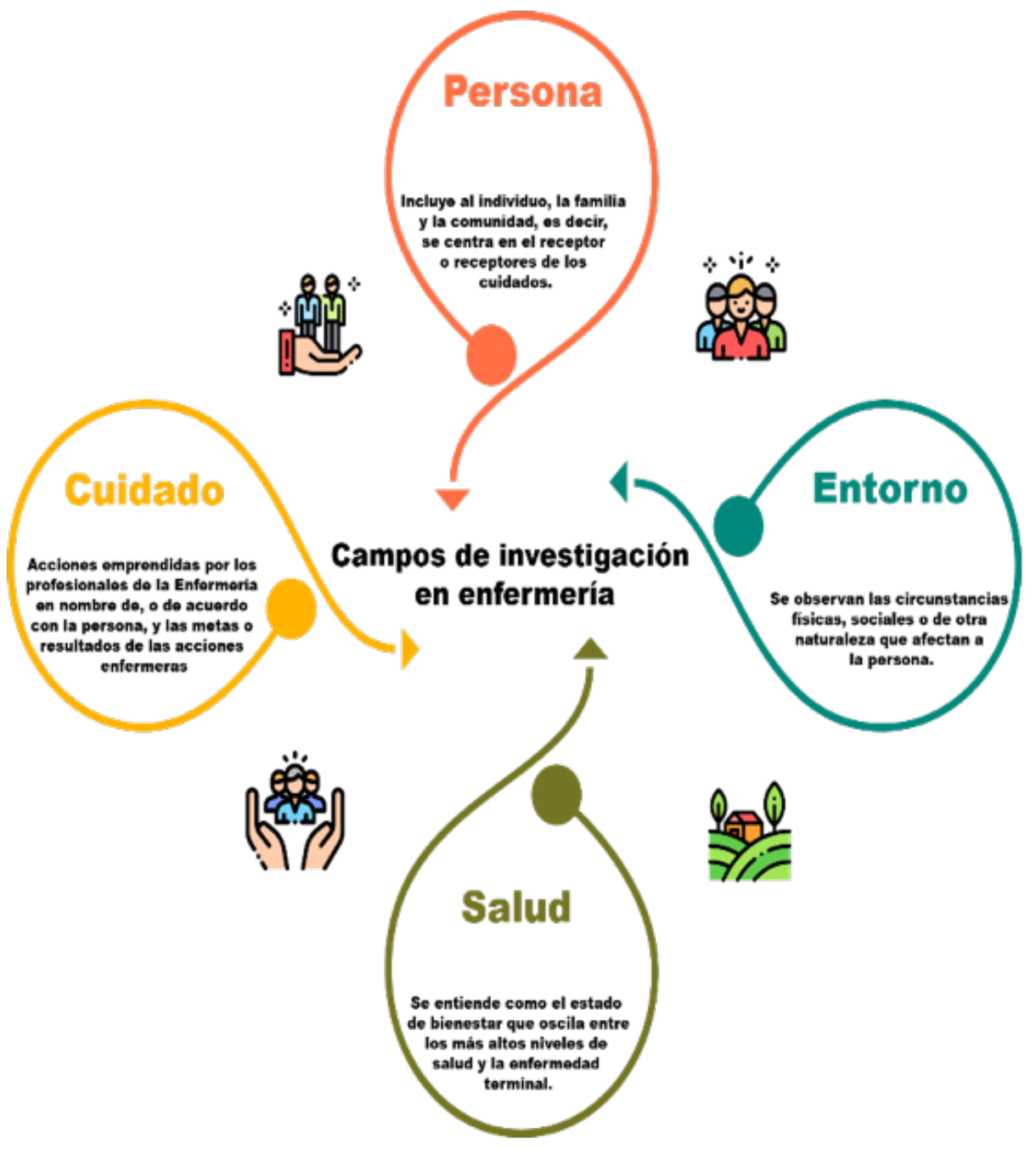


Es a partir de estos campos del quehacer investigativo que se da cuenta de que el enfoque mixto es el ideal para cubrir la investigación en enfermería, ya que requiere de una evidencia que ponga soluciones acertadas en beneficio de los actores sociales, puesto que las problemáticas son complejas. Dicha complejidad, solamente podrá ser abordada con datos medibles e interpretables.

Con la integración de los enfoques que sustentan al enfoque mixto, se podrán explorar distintos niveles del problema de estudio en el desempeño profesional de la enfermería. La ubicación del proceso de investigación, en cada una de sus etapas, promueve obtener una mayor variedad de perspectivas del fenómeno: frecuencia, amplitud y magnitud (cuantitativa), así como profundidad y complejidad (cualitativa), generalización (cuantitativa) y comprensión (cualitativa) [Creswell, 2009].

Por lo tanto, los métodos cuantitativo y cualitativo proporcionan a la investigación en enfermería una visión, "fotografía" o "trozo" amplio de la realidad, forjando el diseño de propuestas de solución a la medida de las necesidades de los grupos metas.

Las etapas en las que suelen integrarse los enfoques cuantitativo y cualitativo son, fundamentalmente, el planteamiento del problema, el diseño de investigación, el muestreo, la recolección de los datos, los procedimientos de análisis y/o interpretación de los datos o resultados (Hernández Sampieri, Fernández Collado y Baptista Lucio, 2014).
Es importante resaltar que el propósito de la investigación mixta no es sustituir a ninguno de los enfoques que la componen, sino que, por el contrario, su meta es utilizar las fortalezas de ambos tipos de indagación, combinándolas y tratando de minimizar sus debilidades potenciales. De esta manera, lograr una perspectiva más amplia y profunda del fenómeno, formular el planteamiento del problema con mayor claridad, así como las maneras más apropiadas para estudiar y teorizar los problemas de investigación, producir datos más 'ricos' y variados mediante la multiplicidad de observaciones, potenciar la creatividad teórica por medio de suficientes procedimientos críticos de valoración, efectuar indagaciones más dinámicas y reforzar con mayor solidez las inferencias científicas, que si se proponen de manera aislada (Lara Muñoz, 2011).

Por ende, la aplicación de este enfoque en la investigación en el área de enfermería requiere que se cuente con equipo entrenado en los enfoques cuantitativos y cualitativos, que sus conocimientos se fortalezcan en el método donde hay poca experticia. Asimismo, que el tiempo por invertir en el proceso sea acorde con un plan de trabajo realista y muy bien estructurado sin perder de vista los principios éticos establecidos. Dentro de las posibles líneas de investigación, se destacan ocho grandes grupos con miras al cumplimiento de los Objetivos de Desarrollo Sostenible de la Organización de las Naciones Unidas (ver figura 4).

De acuerdo con el espíritu del objetivo 3, de 17 objetivos planteados por la $\mathrm{ONU}$, es fundamental garantizar una

Figura 4. Propuestas de líneas de investigación desde la enfermería, con base en el planteamiento de Santos Heredero, Rodríguez Arias, Rodríguez Ballestero, 2004. (Plantilla tomada de freepik.es y adaptada por las autoras como elaboración propia)

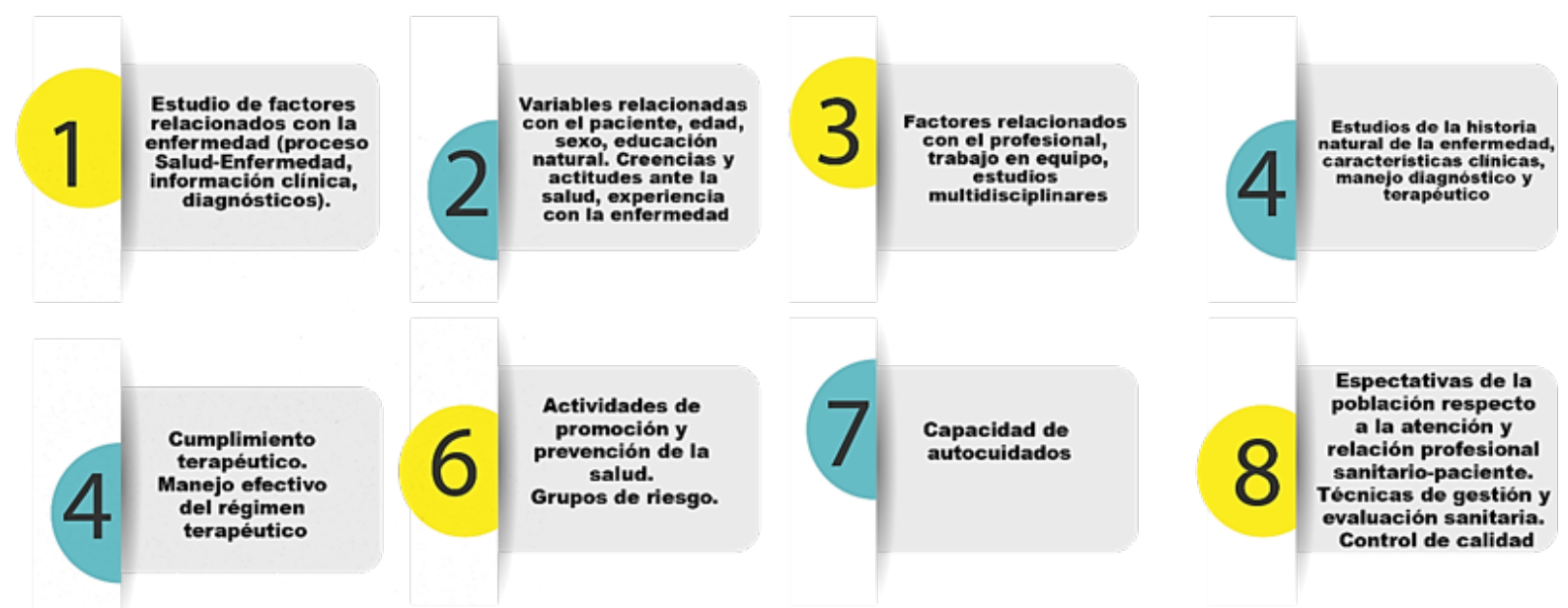


vida saludable y promover el bienestar universal, es decir, para todos en todas las edades. Por lo que se necesitan iniciativas, procedentes de las investigaciones, para erradicar por completo una amplia gama de enfermedades y para hacer frente a numerosas y variadas cuestiones persistentes y emergentes relativas a la salud.

A manera de consideraciones finales, se plantean las siguientes mociones:

1. Cabe decir, tal como lo expresara el científico español Severo Ochoa, que "en principio, la investigación necesita más cabezas que medios",. Así pues, la enfermería como profesión se ha configurado con capacidad y responsabilidad para desarrollar investigaciones científicas que proporcionen la capacidad de resolver necesidades urgentes en el ámbito de la salud a partir de la aplicación de los enfoques que componen la investigación mixta.

2. Que el hecho de que se propongan las líneas de investigación antes mencionadas no significa que se quiera usurpar espacios de otras disciplinas de las ciencias de la salud en particular o de otras áreas del conocimiento. Por el contrario, participar en proyectos de carácter multidisciplinario en los que desde la investigación mixta aporten nuevos conocimientos a problemáticas de la salud muy complejos.

3. El presente artículo solamente es un primer aporte, aproximado, como apertura de la discusión de la temática planteada y para poner de relieve la visibilidad de que en enfermería se hace investigación científica como en cualquier otra área del conocimiento.

\section{Referencias}

Creswell, J. W. (2009). Research design: Qualitative, quantitative, and mixed methods approaches (4a ed.) [versión de Adobe Acrobat Reader]. Recuperado de http://fe.unj.ac.id/wp-content/uploads/2019/08/ Research-Design_Qualitative-Quantitative-and-MixedMethods-Approaches.pdf

De Gortari, E. (1979). Introducción a la lógica dialéctica (5a ed.) [versión de Adobe Acrobat Reader]. Recuperado de https://ymibosquemadura.files.wordpress.com/2015/09/ eli-de-gortari-introduccion-a-la-logica-dialectica.pdf
Hernández Sampieri, R., Fernández Collado, C. y Baptista Lucio, M. P. (2014). Metodología de la investigación (6 ${ }^{a}$ ed.) [versión de Adobe Acrobat Reader]. Recuperado de https://www.uca.ac.cr/wp-content/uploads/2017/10/ Investigacion.pdf

Hurtado de Barrera, J. (2012). Metodología de la Investigación Holística: Guía para la compresión holística de la ciencia ( $4^{\mathrm{a}}$ ed.) [versión de Adobe Acrobat Reader]. Recuperado de https://dariososafoula.files.wordpress.com/2017/01/ hurtado-de-barrera-metodologicc81a-de-lainvestigaciocc81n-guicc81a-para-la-comprensiocc81nholicc81stica-de-la-ciencia.pdf

Izcara Palacios, S. P. (2014). Manual de investigación cualitativa [versión de Adobe Acrobat Reader]. Recuperado de http://www.grupocieg.org/archivos/Izcara\%20(2014)\%20 Manual\%20de\% 20Investigaci\%C3\%B3n\%20Cualitativa.pdf

Lara Muñoz, E. M. (2011). Fundamentos de Investigación. Un enfoque por competencias [versión de Adobe Acrobat Reader]. Recuperado de https://www.academia. edu/38297884/Fundamentos_de_Investigacion-lara

Núñez Tenorio, J. R. (1989). Metodología de las ciencias sociales [versión de Adobe Acrobat Reader]. Recuperado de https://books.google.com.sv/books?id=iN9nL4h65 $5 \mathrm{gC} \& \mathrm{lpg}=\mathrm{PA} 244 \&$ ots=bnSWAehfiU $\& \mathrm{dq}=\mathrm{Nu} \% \mathrm{CC} \% 81 \mathrm{n}$ \%CC\%83ez\%20Tenorio\%2C\%20J.\%20R.\%20(1989).\%20 Metodolog\%C3\%ADa\%20de\%20las\%20ciencias\%20 sociales. $\&$ hl=es\&pg=PA\&\#v=onepage \&q\&f=false

Paensa, A. (2012). Matemática para todos [versión de Adobe Acrobat Reader]. Recuperado de http://cms.dm.uba.ar/ material/paenza/libro7/matematica_para_todos.pdf

Rojas Soriano, R. (2013). Guía para realizar investigaciones sociales [versión de Adobe Acrobat Reader]. Recuperado de https://raulrojassoriano.com/cuallitlanezi/wp-content/ themes/raulrojassoriano/assets/libros/guia-realizarinvestigaciones-sociales-rojas-soriano.pdf

Santos Heredero, F. X., Rodríguez Arias, C. A. y Rodríguez Ballestero, R. (2004). Metodología básica de investigación en enfermería [versión de Adobe Acrobat Reader]. Recuperado de https://kupdf.net/download/metodologia basicadeinvestigacionenenfermeria_59e7cf4f08bbc5aa1 8e65383_pdf 